\title{
Move - \\ Das Zusammenspiel von Bewegtem und Bewegendem
}

\author{
Melanie Mack \\ Universität Leipzig \\ Institut für Bewegungs- und Trainingswissenschaft
}

Der Begriff der Bewegung ist in allen Wissenschaften zu finden: als soziale Bewegung durch das Verhalten von kollektiven Akteuren; in körperlicher Aktivität oder dem Sport als physische Bewegung; bei Zugvögeln, die im Herbst Europa verlassen, um im Süden zu überwintern; oder in Gletscherbewegungen infolge der Gravitation. Die genannten Szenarien sind komplexe Systeme, sie enthalten mehrere Komponenten, die miteinander interagieren. Ändert oder bewegt sich eine Komponente, hat das oft Folgen für das gesamte System. Wenn wir etwa unsere Körper zur Musik bewegen, dann verursachen eine Vielzahl an Variablen, welche den unterschiedlichsten Wissenschaftsrichtungen zugeordnet werden können, unsere Tanzbewegungen: die Musik (Musikwissenschaft), unsere biomechanischen Eigenschaften (Sportwissenschaft), unsere Vorerfahrung (Psychologie), etc. Im Forschungsprozess ist es darum bei vielen Fragestellungen essenziell, interdisziplinär zusammenzuarbeiten, oder zumindest einen Blick über den Tellerrand zu wagen.

In der vorliegende Ausgabe von arbeitstitel - Forum für Leipziger Promovierende setzten sich NachwuchswissenschaftlerInnen interdisziplinär mit diversen Aspekten von Bewegung auseinander. Was wird unter Bewegung verstanden, wo ist Bewegung $\mathrm{zu}$ finden, wie verändert sich Bewegung und wie nehmen wir sie wahr? Das Themenspektrum der Beiträge ist breit und vielfältig.

Raphael Borchers erläuterte die leibnizsche Reflexion der Bewegung und ihre Bedeutung für die Herausbildung moderner Wissensdisziplinen im 17. Jahrhundert anhand eines kleinen Ausflugs in die Begriffs- und Wissenschaftsgeschichte. Florian Teichmann untersuchte aus einer kognitionspsychologischen Perspektive das Bewegungslernen und dabei spezifisch den Zusammenhang zwischen Körperkontrolle und Körperwahrnehmung. Auch im Beitrag von Antje Goller ist Bewegung mit Lernen assoziiert. Die Erziehungswissenschaftlerin beschäftigte sich mit der Frage, wie Nachhaltigkeit in Lehr- und Lernsituationen thematisiert werden kann, ohne dabei zu überwältigen oder zu moralisieren. Jana Raffels Beitrag erörtert Rollen und Interaktionsmuster ägyptischer Gottheiten in Heilsprüchen und Tobias Neidel tauchte in den Kontext des staatlichen Sicherheitsgefüges in Deutschland ein. Der Autor versteht dieses hierbei als dynamisches Arrangement verschiedener Gruppierungen und Akteuren, welches einem ständigen Wandel und struktu-

Erschienen in: arbeitstitel - Forum für Leipziger Promovierende, Band 8, Heft 1 (2020), S. 1. Lizenzierung CC-BY-SA 4.0. rellen Anpassungen unterworfen ist. In die Politikwissenschaft geht es bei dem Beitrag von Florian Spissinger: der Autor beschäftigte sich mit der Bewegtheit von Affekten und untersuchte dabei, wie Affekte „on the move“ in politischen Arenen eingesetzt werden. Mit der Thematik wie Bewegung in der Musik beschrieben und wahrgenommen wird beschäftigte sich Nina Julich-Warpakowski. Sie setzt sich hierbei mit der Fragestellung auseinander, inwiefern musikalische Bewegungsausdrücke als wenig metaphorisch empfunden werden.

Wir, die gesamten Herausgeberschaft von arbeitstitel Forum für Leipziger Promovierende, freuen uns, dass Bewegung aus so vielen unterschiedlichen Perspektiven untersucht und erörtert wurde und hoffen, dass der Blick über den Tellerrand der eigenen Disziplin etwas in Euch bewegt hat. Manchmal sind es auch nur die kleinen, die unsichtbaren Dinge, die uns bewegen und die dadurch die Welt bewegen. Und wenn noch nicht heute, dann möglicherweise morgen! Wir danken allen, die bei dieser Ausgabe mitgewirkt haben: dem PromovierendenRat der Universität Leipzig für ihr Engagement und die Übertragung der Verantwortlichkeit; der Research Academy für die Bereitstellung der Räumlichkeiten für die Vorträge; dem Open Science Office der Universität Leipzig für die Bereitstellung der digitalen Plattform; den GutachterInnern Anne-Coralie Bonnaire, Dorothea Heilmann, David Georg Reichelt, Robert Reimer, Benjamin Reimann, Moritz Rudolph, Thomas Schmid für ihr konstruktives Feedback. Nicht zuletzt danken wir natürlich vor allem den NachwuchswissenschaftlerInnen für ihre Beiträge - und wünschen ihnen viel Erfolg für die Zukunft!

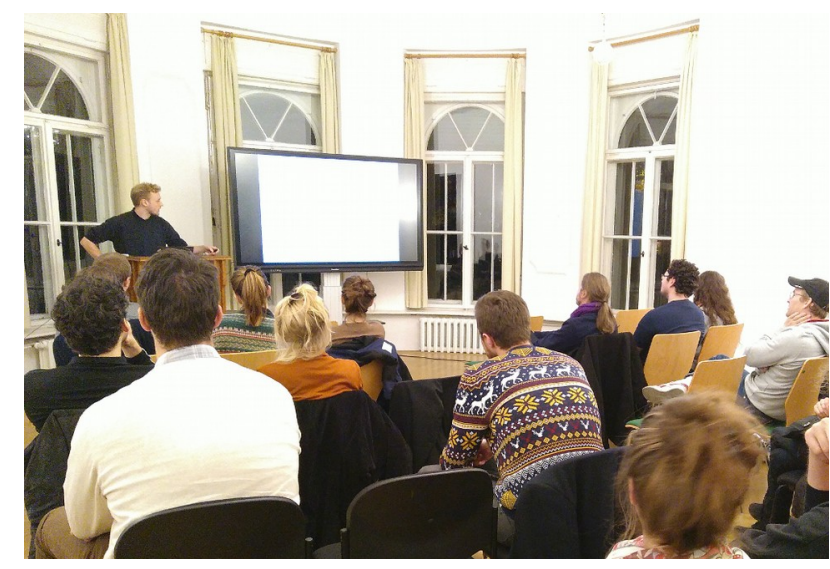

Abbildung 1. Impressionen von den 22. Leipziger Promotionsvorträgen am 22. November 2019. (Foto: Anne-Coralie Bonnaire). 